\title{
Sources of noise in Brillouin optical time-domain analyzers
}

\author{
Javier Urricelqui ${ }^{1,2}$, Marcelo A. Soto ${ }^{1, *}$, Luc Thévenaz ${ }^{1}$ \\ ${ }^{1}$ EPFL Swiss Federal Institute of Technology, Institute of Electrical Engineering, \\ SCI STI LT, Station 11, CH-1015 Lausanne, Switzerland \\ ${ }^{2}$ Universidad Pública de Navarra, Campus Arrosadia s/n, 31006 Pamplona, Spain \\ *E-mail: marcelo.soto@epfl.ch
}

\begin{abstract}
This paper presents a thorough study of the different sources of noise affecting Brillouin optical time-domain analyzers (BOTDA), providing a deep insight into the understanding of the fundamental limitations of this kind of sensors. Analytical and experimental results indicate that the noise source ultimately fixing the sensor performance depends basically on the fiber length and the input pump-probe powers. Thus, while the phase-to-intensity noise conversion induced by stimulated Brillouin scattering can have a dominating effect at short distances, a combination of sources determines the noise in longrange sensing, basically dominated by probe double Rayleigh scattering.
\end{abstract}

Keywords: Optical fiber sensor, stimulated Brillouin scattering, distributed fiber sensor, intensity noise

\section{INTRODUCTION}

During the last decades, Brillouin optical time domain analysis (BOTDA) sensors have been the focus of intense research activities, essentially with the purpose of enhancing the sensor performance. A recent study ${ }^{1}$ has established that the signal to noise ratio (SNR) of the measurements is the key scaling factor that defines the ultimate performance of a BOTDA sensor ${ }^{1}$. This actually depends on different parameters, such as the sensing distance, the spatial resolution, the maximum pump and probe levels coupled into the fiber (limited by nonlinear effects) and the noise level affecting the measurements. The limitations imposed by all these factors, except noise, have been vastly characterized and modeled in the state-of-theart. Recently, there have been some preliminary studies addressing the impact of phase $\mathrm{e}^{2}$ and intensity ${ }^{3}$ noise in Brillouinbased systems. They indicate that the phase noise of the laser could be relevant in a pump-probe interaction using two continuous-waves $(\mathrm{CW})^{2}$, while the probe relative intensity noise (RIN) has a dominating impact on the ultimate performance of optimized BOTDA systems ${ }^{3}$.

In this work, the different noise sources existing in a BOTDA sensor are theoretically and experimentally analyzed. The study points out that, under optimized pump-probe power conditions, the probe RIN resulting from the phase-to-intensity conversion occurring in stimulated Brillouin scattering (SBS) and in the probe double Rayleigh scattering (DRS) are two of the most relevant sources of noise limiting the performance of the conventional BOTDA sensing technique.

\section{NOISE ADDED TO THE PROBE DURING PROPAGATION}

The noise present in a BOTDA sensor has been experimentally studied using a conventional scheme (shown in Fig. 1) ${ }^{1}$. In order to have a representative condition and an optimized SNR, a $125 \mathrm{MHz}$ photo-detector with a high trans-impedance amplifier has been used. Under this condition the voltage amplitude related to thermal noise would dominate compared to the shot noise; however, as it will be shown later, other sources of noise can still have a dominating effect depending on the input pump and probe powers. The impact of the laser phase noise is analyzed using two lasers: a distributed feedback (DFB) laser of $1.6 \mathrm{MHz}$ linewidth and an external cavity (EC) laser of $20 \mathrm{kHz}$ linewidth. To study the phase-to-intensity noise conversion occurring in the sensing fiber, the fiber length has been varied from a few meters up to several tens of $\mathrm{km}$.

\subsection{Noise spectral power density along the probe propagation path}

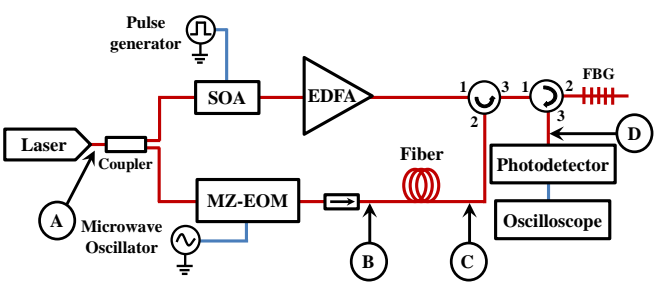

The first part of the study focuses on the noise generated along the Figure 1. Experimental setup. MZ-EOM: Mach propagation path of the probe. Using an electrical spectrum analyzer Zehnder electro-optic modulator, EDFA: Erbium(ESA) connected to a $125 \mathrm{MHz}$ photo-detector, the accumulated noise doped fiber amplifier, SOA: Semiconductor optical along the probe path has been measured at different points (labeled as A- amplifier, FBG: fiber Bragg grating. 
D in Fig. 1), with no pump pulse propagating in the fiber. Using a variable optical attenuator, the input power of the photo-detector has been set to $-18 \mathrm{dBm}$ for each measurement, ensuring a known shot noise level. Fig. 2 shows the electrical power spectral density (PSD) measured in a $50 \mathrm{~km}$-long BOTDA sensor. The black curve is the thermal noise of the detector (equivalent to a noise amplitude $\sigma_{t h}=890 \mu \mathrm{V}$ ). Note that the noise spectrum is not flat since the PSDs also contain the band-pass response of the electrical amplification stages inside the receiver. The green curve is the PSD measured when detecting the laser output, i.e. at point A in Fig. 1. It was verified that this spectrum corresponds mainly to the shot noise contribution $\left(\sigma_{s h}=464.4 \mu \mathrm{V}\right)$ added to the thermal noise, and the laser RIN $(<150 \mathrm{~dB} / \mathrm{Hz}$ ) has no observable effect on the measurements. To evaluate the noise introduced by the generation of the 2-sideband probe, the PSD at the EOM output has been measured (point B in Fig. 1). Results indicate that the PSD at point $B$ is the same as the one at point $A$, proving that no noise is

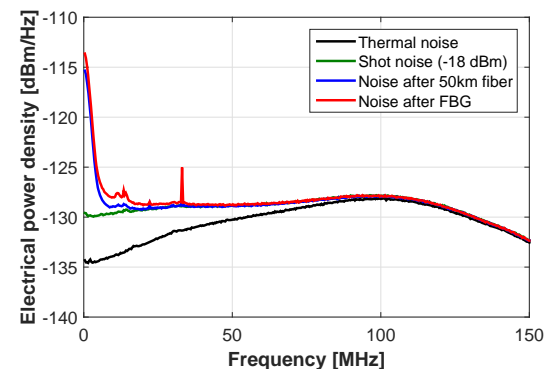

Figure 2. PSD measured at different points in the system. Black and green curves are the thermal and shot noise of the detector; blue and red curves are the PSD after the fiber and after the FBG.

added by the modulation. Then, the noise PSD measured at the sensing fiber output (point $C$ ) shows that low frequency components increase significantly (blue curve). As it will be described later, this is due to phase-to-intensity noise conversion due to double Rayleigh scattering of the probe. Finally, the red curve shows the noise PSD at point D, and include the phase-to-intensity noise conversion taking place in the FBG at the receiver. Note that curves show a peak at $33 \mathrm{MHz}$; however, this is related to a component introduced by the laser driver, and has no relevance for the study.

\subsection{Double Rayleigh scattering generated by the probe wave}

Double Rayleigh scattering corresponds to a multi-path interference that converts signal phase noise into intensity noise ${ }^{4}$. In the case of BOTDA sensors, the probe generates DRS along the sensing fiber. Unfortunately the probe DRS components propagate in the same band as the useful probe signal and thus, they cannot be eliminated by simple filtering. The noise PSD due to DRS can be expressed as ${ }^{4}$ :

$$
P_{D R S}(f)=\frac{10}{9} R_{R b}^{2}\left(2 \alpha L+e^{-2 \alpha L}-1\right) \cdot S_{\text {laser }}(f)
$$

where the constant takes into account the fiber birefringence, $R_{R b}$ is the Rayleigh backscattering coefficient, $\alpha$ is the fiber loss, $L$ is the fiber length and $S_{\text {laser }}(f)$ is the spectrum of the optical signal. Fig. 3(a) compares the measured DRS noise PSD (marker points) with the respective theoretical PSD (continuous lines) obtained by Eq. (1), for standard single-mode fibers (SMFs) of different lengths. Theoretical and experimental curves, both obtained with a DFB laser, show a good agreement, demonstrating that the noise induced in the fiber is essentially due to probe DRS. In contrast to fiber nonlinearities, the DRS power does not depend on the fiber effective length, but grows linearly with distance. This behavior actually makes the DRS-induced noise to be very important in long-range BOTDA sensing, becoming one of the dominating sources of noise. This is especially critical in sensors that use a leading fiber to propagate the probe to a remote $\operatorname{location}^{5}$, since this signal has to propagate along a fiber two-fold longer than the actual sensing fiber.
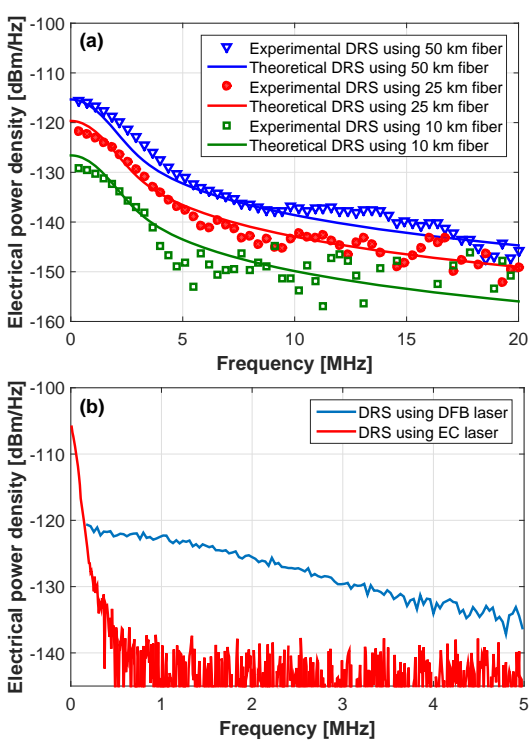

Figure 3. Spectral power density of the probe DRS. (a) Effect of the fiber length, using a DFB laser, and (b) effect of the laser linewidth (1.6 MHz vs $20 \mathrm{kHz}$ )

To analyze the effects of the laser phase noise, the DRS-induced noise measured after a $25 \mathrm{~km}$-long fiber with a DFB laser and an EC laser (having the same power) are compared in Fig. 3(b). Although the PSD narrows for the EC laser, the spectral components within the laser linewidth become stronger, leading to the same total noise amplitude: $\sigma_{D R S}=247.2 \mu \mathrm{V}$ for the DFB laser and $\sigma_{D R S}=249.2 \mu \mathrm{V}$ for the EC laser.

\subsection{Phase-to-intensity noise conversion in FBG}

Conventional BOTDA sensors use narrowband FBGs to select the probe signal to be detected. Unfortunately, the reflection band of FBGs is temperature dependent, and therefore, it can likely shift during operating conditions. If the probe signal is tuned at the top of a flat FBG, there should be no significant noise added to the probe. However, when the probe optical frequency lies on the slope of the FBG, the laser phase noise is converted into intensity noise ${ }^{6}$. To verify this, the standard deviation of the photo-detector voltage has been measured as a function of wavelength within the FBG reflection band. While Fig. 4(a) depicts the spectrum of the top region of the FBG reflection, Fig. 4(b) shows the FBG-induced noise 
amplitude. It can be seen that this noise increases in the slopes of the FBG reflection spectrum (up to $\sigma_{F B G}=\sim 3.5 \mathrm{mV}$ in this case). However, in a welltuned condition the FBG-induced noise should be expected to be lower than the other noise levels reported in this paper.

\section{NOISE INDUCED BY PUMP-PROBE INTERACTION}

The sources of noise analyzed so far consider only the propagation of the probe along the system. When pump pulses travel in the fiber new sources of noise appear. One of them is the Rayleigh scattering originated by the pump; however, this can be easily eliminated using suitable optical filtering. In addition, the pump RIN can be generally neglected owing to the low cut-off frequency of the pump-probe RIN transfer in the SBS process ${ }^{7}$. More
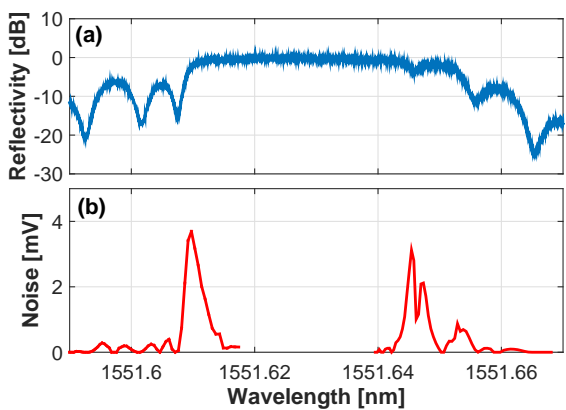

Figure 4. Measured (a) FBG reflectivity and (b) FBG-induced noise amplitude. important are: $i$ ) the noise generated by phase-to-intensity noise conversion in the SBS process, and ii) the noise resulting from the pump-induced spontaneous Brillouin scattering (SpBS) beating with the probe at the receiver. These two sources of noise are described below. In order to obtain a reliable characterization of them, and to fully mitigate the effect of polarization fluctuations inside the sensing fiber, a $90 \mathrm{~m}$-long polarization-maintaining fiber has been used in this case. Note that the noise induced by polarization fluctuations in a standard SMF highly depends on the polarization diversity method and can be simply minimized by a proper control of the input pump and probe polarization states.

\subsection{Phase-to-intensity noise conversion in the SBS process}

In standard BOTDA schemes, pump and probe interact through SBS at each fiber position, activating a local acoustic wave for a few tens of ns. This short activation time is generally much shorter than the coherence time of the laser (typically related to linewidths of a few $\mathrm{MHz}$ ). Unlike the CW case ${ }^{2}$, the acoustic wave is once activated with highly coherent light, resulting in a negligible influence from the laser phase noise. However, a large number traces is typically averaged to increase the SNR of the measurements. As a result of the laser spectral positioning uncertainty due to phase noise, the gain experienced by the probe wave at a given fiber position is slightly different for each trace acquisition, inducing a phaseto-intensity noise conversion given by:

$$
\sigma_{S B S}(\Delta v)=2 \Delta v_{L} \cdot G_{S B S}^{\prime}(\Delta v)
$$

where $\Delta v_{L}$ is the laser linewidth, $G_{S B S}^{\prime}(\Delta v)$ is the frequency derivative of the Brillouin gain spectrum (BGS) at a given pump-probe detuning $\Delta v$. Fig. 5(a) compares the measured noise (continuous line) as a function of the frequency detuning using a DFB laser and the theoretical noise amplitude obtained by Eq. (2) (dashed line), for pulses of $10 \mathrm{~ns}$ and 70 ns (for a fair comparison, the pump power has been adjusted to have the same Brillouin gain in the two cases). Note that, at the
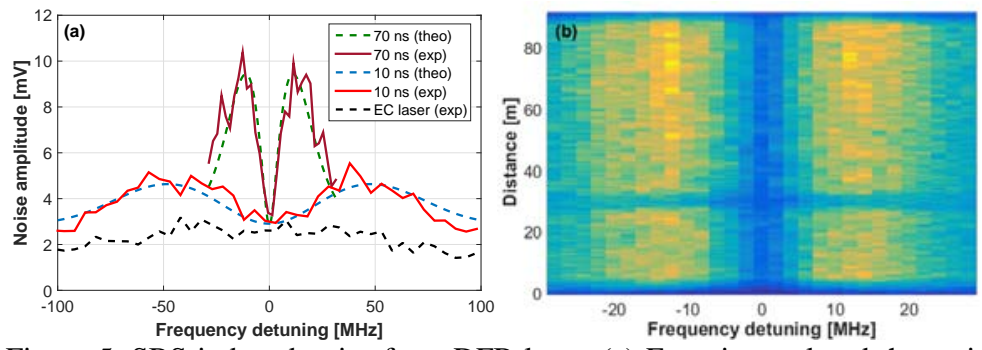

Figure 5. SBS-induced noise for a DFB laser. (a) Experimental and theoretical noise amplitude at the end of the fiber are compared for $10 \mathrm{~ns}$ and $70 \mathrm{~ns}$ pump pulses. The black dashed line is the noise measured with an EC laser. (a) Topview of 3D noise map distance vs frequency detuning for a 70 ns pump pulse.

Brillouin peak frequency (zero detuning), a minimum noise level is induced due to the zero gradient of the BGS; however, a maximum phase-to-intensity conversion occurs at the BGS full-width half-maximum (100 MHz and $35 \mathrm{MHz}$ for the $10 \mathrm{~ns}$ and $70 \mathrm{~ns}$ pulse, respectively), where the BGS slope is maximum. It is worth noticing that the minimum noise amplitude matches the level measured with the EC laser (black dashed line). Due to the reduced linewidth of the EC laser, the SBS-induced phase-to-intensity noise conversion is negligible; and therefore the noise level shown in the figure corresponds the SpBS beating with the probe at the receiver (this will be described below). Fig. 5(b) shows the top-view of the 3D map of the detected noise versus distance and frequency detuning. Note that, at $30 \mathrm{~m}$ from one of the fiber inputs, the SBS-induced noise amplitude vanishes as a result of the high phase correlation between pump and probe at this position (this actually corresponds to the fiber location at which pump and probe have propagated the same distance from the laser, so that their phases are perfectly correlated).

\subsection{SpBS-probe beating noise}

When the pump pulse propagates in the fiber, spontaneous Brillouin scattering is generated at each location, at a frequency that depends on the local Brillouin frequency shift (BFS). Since the receiver stage is tuned to detect the probe scanning the 
BGS, both probe and SpBS reach the photodetector, giving rise to a beating noise that depends on the detector bandwidth and the detuning between the pump-probe frequency offset and the BFS. Fig. 6 shows the PSD measured for a detuning ranging from $0 \mathrm{MHz}$ up to $140 \mathrm{MHz}$, with $10 \mathrm{MHz}$ steps, when using an EC laser (Fig. 4(a)) and a DFB laser (Fig. 4(b)). Curves show how the frequency content of the beating noise shifts towards higher frequencies when the detuning
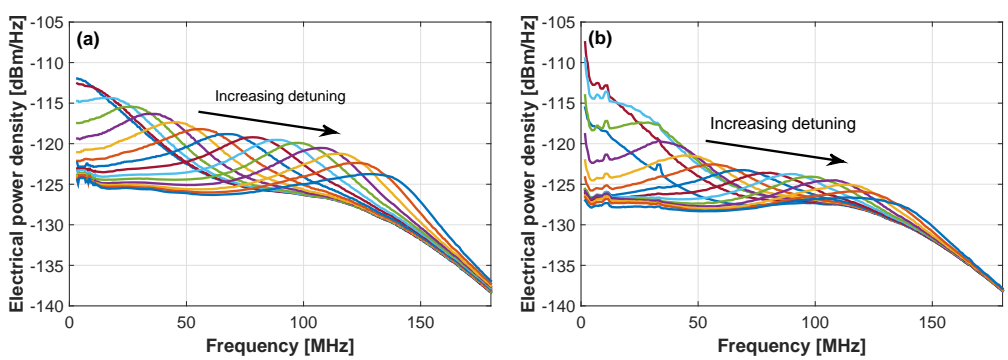

Figure 6. PSD of beating between probe and pump-induced SpBS. For (a) an EC and (b) a DFB laser. Measurements for different detuning, with $10 \mathrm{MHz}$ steps. increases. Since measurements in Fig. 6 are obtained while pump and probe counter-propagate and interact through SBS, the PSDs also include the effect of phase noise on the SBS process. Thus, while Fig. 4(a) shows essentially the SpBSprobe beating noise with negligible SBS-induced noise; Fig. 4(b) shows that the level obtained with the DFB laser at zerodetuning (blue curve having the maximum at zero frequency) is lower than the noise measured in the BGS slope, following the same behavior described in Fig. 5. The SpBS beating noise is evaluated by integrating each curve (removing the contribution of the SBS-induced noise and the spectral response of the receiver), giving an average frequency-independent amplitude $\sigma_{S p B S}=\sim 2.5 \mathrm{mV}$, which agrees with the black dashed line in Fig. 5(a).

\section{CONCLUSION}

The different noise sources existing in BOTDA sensors have been analyzed and modeled. Results point out that under optimized receiver conditions, i.e. having a high trans-impedance to improve the SNR, the dominating noise is highly dependent on the sensing range of the system. Thus, in short distance scenarios (less than about $15 \mathrm{~km}$ ), where the Brillouin gain is relatively high and the pump power has not been highly attenuated ( $<3 \mathrm{~dB}$ loss), the phase-to-intensity noise conversion in the SBS process and the SpBS-probe beating noise are the dominating sources of noise in the system. When the sensing range increases, the Brillouin gain experienced by the probe wave and the SpBS components backscattered from the far end of the fiber are reduced due to the fiber attenuation, and hence, thermal noise could have a dominating contribution. However, the DRS-induced noise also plays an important role since it increases linearly with the fiber length and can become the dominating source of noise at very long sensing ranges ( $>50-70 \mathrm{~km}$ ), especially if an additional leading fiber is used to propagate the probe wave to a very remote location (thus doubling both the probe propagation length and the DRS-induced noise). It is also important to mention that in dynamic sensing, based for instance on a slopeassisted BOTDA scheme, where the probe wave is tuned to the wings of the Brillouin gain spectrum, the SBS-induced phase-to-intensity noise conversion increases considerably and therefore it may induce a significant degradation of the SNR and sensor performance.

J. Urricelqui acknowledges the support from the Spanish Ministerio de Economia y Competitividad through the project TEC2013-47264-C2-2-R, FEDER funds, the COST action TD1001 and Universidad Pública de Navarra. M. A. Soto and L. Thévenaz acknowledge the support from the Swiss Commission for Technology and Innovation (Project 13122.1).

\section{REFERENCES}

[1] Soto, M. A. and Thévenaz, L., "Modeling and evaluating the performance of Brillouin distributed optical fiber sensors," Opt. Express 21(25), 31347-31366 (2013).

[2] Shlomovits, O., Langer, T., and Tur, M., "Source-induced noise in stimulated Brillouin scattering”. Proc. SPIE 9157, 23rd International Conference on Optical Fibre Sensors, 915772 (June 2, 2014).

[3] Soto, M. A. and Thévenaz, L., “Towards 1’000’000 resolved points in a distributed optical fibre sensor”. Proc. SPIE 9157, 23rd International Conference on Optical Fibre Sensors, 9157C3 (June 2, 2014).

[4] Wan, P., and Conradi, J., "Impact of double Rayleigh backscatter noise on digital and analog fiber systems," J. Lightwave Technol. 14(3), 288-297 (1996)

[5] Soto, M. A., et al., "Extending the Real Remoteness of Long-Range Brillouin Optical Time-Domain Fiber Analyzers,” J. Lightwave Technol. 32(1), 152-162 (2014).

[6] Liu, Q., Tokunaga, T., and He, Z., "Realization of nano static strain sensing with fiber Bragg gratings interrogated by narrow linewidth tunable lasers,” Opt. Express 19(21), 20214-20223 (2011).

[7] Zhou, J., Chen, J., Jaouen, Y., Yi, L., Petit, H., and Gallion, P., “A New Frequency Model for Pump-to-Signal RIN Transfer in Brillouin Fiber Amplifiers,” IEEE Photon. Technol. Lett. 19(13), 978-980 (2007). 